\title{
Numerical Estimation of Spectral Properties of Laser Based on Rate Equations
}

\author{
Jan Litvik, ${ }^{1}$ Michal Kuba, ${ }^{1}$ Daniel Benedikovic, ${ }^{2}$ Jozef Dubovan, ${ }^{1}$ and Milan Dado ${ }^{1}$ \\ ${ }^{1}$ Faculty of Electrical Engineering, University of Zilina, Univerzitna 1, 01026 Zilina, Slovakia \\ ${ }^{2}$ Centre for Nanoscience and Nanotechnology, CNRS, Université Paris-Sud, Université Paris-Saclay, C2N-Orsay, \\ 91405 Orsay Cedex, France \\ Correspondence should be addressed to Jan Litvik; jan.litvik@fel.uniza.sk
}

Received 27 July 2016; Accepted 26 October 2016

Academic Editor: Luciano Mescia

Copyright (C) 2016 Jan Litvik et al. This is an open access article distributed under the Creative Commons Attribution License, which permits unrestricted use, distribution, and reproduction in any medium, provided the original work is properly cited.

\begin{abstract}
Laser spectral properties are essential to evaluate the performance of optical communication systems. In general, the power spectral density of the phase noise has a crucial impact on spectral properties of the unmodulated laser signal. Here the white Gaussian noise and $1 / f$-noise are taken into the consideration. By utilizing the time-dependent realizations of the instantaneous optical power and the phase simultaneously, it is possible to estimate the power spectral density or alternatively the power spectrum of an unmodulated laser signal shifted to the baseband and thus estimate the laser linewidth. In this work, we report on the theoretical approach to analyse unmodulated real-valued high-frequency stationary random passband signal of laser, followed by presenting the numerical model of the distributed feedback laser to emulate the time-dependent optical power and the instantaneous phase, as two important time domain laser attributes. The laser model is based on numerical solving the rate equations using fourthorder Runge-Kutta method. This way, we show the direct estimation of the power spectral density and the laser linewidth, when time-dependent laser characteristics are known.
\end{abstract}

\section{Introduction}

Constant growth in transmission data and capacity leads to the deployment of new optical technologies that are used in the infrastructure of communication systems. Recently, the emphasis is devoted to the transmission systems with capacity in excess $100 \mathrm{~Gb} \cdot \mathrm{s}^{-1}$ per one channel. This yields to the overall capacity of the optical communication systems ranging from $10 \mathrm{~Tb} \cdot \mathrm{s}^{-1}$ to $100 \mathrm{~Tb} \cdot \mathrm{s}^{-1}$ [1]. These levels of the capacity can only be reached by utilizing and combining various optical technologies such as wavelength division multiplexing (WDM), polarization-division multiplexing (PDM), spatial division multiplexing (SDM), optical coherent systems with high-order modulation formats, and digital signal processing (DSP) [1-5].

However, the aforementioned progressive optical technologies desire high-quality sources with a spectral linewidth in order of kilohertz's [1], with high degree of coherence, and low noise level. The optical systems that are able to use advanced modulation formats, for instance, 16-state quadrature amplitude modulation (16-QAM), require laser linewidth of around $1 \mathrm{MHz}$ for symbol rate $10 \mathrm{GBaud}[1$, 6]. In case of quadrature phase shift keying (QPSK), laser linewidths of $10 \mathrm{MHz}$ are usually sufficient $[1,7]$.

The laser phase noise is one of the critical parameters, having a substantial influence on the performance of optical coherent systems, utilizing WDM and high-order modulation formats [8]. Here, the prime goal is to estimate the laser characteristics. Lasers employed in the optical coherent systems are usually used two times in the transmission link: as signal sources at the side of optical transceiver and at the side of optical receiver as local oscillators [8-10].

In the laser active region placed in the cavity, there exists two dominant processes of light generation. First one is the spontaneous emission process, arising from the direct recombination. The second one is light generation caused by the stimulated emission. The recombination process requires the flow of the input optical power. From this reason the stimulated emission leads to the amplification of the incoming photon flux [11]. In general, the laser linewidth 
is considered as a consequence of phase fluctuation in the optical field. The main reason of these fluctuations is the effect of spontaneous emission. This randomly changes the phase and the amplitude of the lasing optical field. The second reason is due to the instantaneous changes the charge carrier density. These variations have an impact on the change of refractive index in the active region of the laser. During the relaxation period oscillations, the change of refractive index causes additional phase shift of the laser field and also leads to the broadening of the laser linewidth [12].

The effect of the laser spectral linewidth broadening can be described by the well-known Schawlow-Townes formula [13]

$$
\delta f=\frac{\pi h f_{0} n_{s p}}{P_{0}\left(2 \pi \tau_{p}\right)}\left(1+\alpha^{2}\right),
$$

where $f_{0}$ is the frequency of lasing light, $h$ is Planck's constant, $\tau_{p}$ is the photon lifetime, $n_{s p}$ is the spontaneous emission factor, $\alpha$ is the linewidth enhancement factor, and $P_{0}$ is the total lasing power. From (1), it becomes apparent that three approaches can be used to reduce the laser spectral linewidth.

First is to increase the photon lifetime by using longer resonator cavity or by improving the reflectivity of the facet. Second approach is to reduce the linewidth enhancement factor by using the negative wavelength detuning of the distributed feedback (DFB) laser or by using the quantum well structures. The third option is to increase the laser output power $[13,14]$.

From (1), it is clear that the laser linewidth is inversely proportional to the output power $P_{0}$. However, the laser linewidth cannot reach zero due to some residual noise of the source. The spectrum of the frequency modulated (FM) noise of the semiconductor lasers is characterized by white frequency noise $[13,15]$. The white frequency noise is primarily caused by the spontaneous emission and the $1 / f$-noise. This quantity is also inversely proportional to the output power. The $1 / f$-noise is almost independent of the output power and it is independent of the time of signal observation [13]. If the white frequency noise is sufficiently reduced by increasing output optical power, the spectral linewidth becomes constant. Experimental results have shown that origin of this residual laser linewidth is caused by flicker $(1 / f)$ noise, which can be observed on the frequencies lower than $100 \mathrm{kHz}[8,11,13]$.

Spectral properties of a laser are determined via spectral properties of the phase noise. It follows that spectral properties of the unmodulated laser signal are naturally determined by power spectral density (PSD) of phase noise [13]. In the literature, these spectral characteristics of laser are typically described by using linewidth. The spectral linewidth of the laser is the only parameter of the PSD and it is characterized as full width at half maximum (FWHM). This parameter is used to comparing different sources of radiation. On the other hand, the linewidth parameter does not provide in-depth information about laser spectral properties. The approach to describe laser characteristics by using the power spectral density of phase noise provides complex information about the laser spectral properties [16-18]. The laser spectrum approximation by Voigt probability distribution allows inclusion of the essential contributions of the white phase noise and $1 / f$-phase noise simultaneously. The white phase noise has a substantial influence on a spectrum shape according to the Cauchy-Lorentz probability density function. The $1 / f$ noise causes spectrum shaping according to the Gaussian probability density function [13]. The Rice probability density function is additional option to approximate the laser spectrum shape, assuming that the laser phase noise has white Gaussian noise character. However, the methods are limited by the attainable bandwidth and the number of samples used in numerical evaluation. By taking this into consideration, the laser spectral PSD can be directly modelled by Rice distribution $[18,19]$. In Section 2, we describe the derivation of the laser PSD and linewidth formulas. The derivation of theoretical laser PSD formulas has been stated in $[13,20]$. In this work, we have performed a revision of this theoretical analysis. The theoretical analysis has been extended in order to obtain relationships in more compact form. Subsequently, it is possible to use the approximation of PSD by Voigt profile for particular laser type, which is given through the numerical model in the form of laser rate equations. The analysis begins from the description of the laser signal as a real-valued random passband signal. The phase fluctuations of the laser signal are considered as a stationary Gaussian random process. By using the autocorrelation function, the laser linewidth is expressed for two theoretical cases. In the first case, the shape of linewidth is approximated by Gaussian shape, while in the second case the Lorentzian shape is considered. In Section 3, we show the application of the presented theoretical approach and its implementation for particular PSD calculation. At the end, we show that the resulting shape of the laser PSD is given by combination of the Gaussian and Lorentzian shape, yielding the Voigt profile. Finally, conclusions are drawn in Section 4.

\section{Derivation of the PSD and Linewidth Formulas}

We consider the unmodulated signal of the laser as a realvalued random passband signal $E(t)$, which can be mathematically expressed as

$$
\begin{aligned}
E(t) & =\left|E_{l}(t)\right| \cos \left[2 \pi f_{0} t+\theta_{E}(t)\right] \\
& =\operatorname{Re}\left\{E_{l}(t) e^{j 2 \pi f_{0} t}\right\}, \quad-\infty<t<\infty,
\end{aligned}
$$

where $E(t)$ is the arbitrary realization of the random laser signal, $E_{l}(t)$ is its complex envelope, that is, complex-valued baseband signal, $t$ is the time, and $f_{0}$ is the reference frequency of the signal $E(t)$. The overall spectrum of the signal $E(t)$ has a symmetrical distribution around the frequency $f_{0}$. Furthermore, $E(t)$ has a constant envelope $\left|E_{l}(t)\right|$ and its random character is determined by the random phase variations $\theta_{E}(t)$ as an argument of its complex envelope [12, $13,15,16]$. Thus, the complex envelope $E_{l}(t)$ of the signal $E(t)$ can be expressed as

$$
E_{l}(t)=\sqrt{2 P_{0}} e^{j \theta_{E}(t)},
$$


where $P_{0}$ is the constant average power of the laser and $\theta_{E}(t)$ is the instantaneous phase of the signal $E(t)$. We expect that the instantaneous phase is stationary Gaussian random process $[14,21,22]$. For the instantaneous phase, it is valid that

$$
\theta_{E}(t)=2 \pi \int_{-\infty}^{t} \nu(\alpha) d \alpha,
$$

where $v(t)$ is the function of instantaneous frequency fluctuations of signal $E(t)$ from mean frequency $f_{0}$ (hereafter abbreviated as phase noise). The instantaneous frequency of the signal $E(t)$ is then $f_{0}+\nu(t)$. Since $\theta_{E}(t)$ is Gaussian random process and according to (4), the quantity $\nu(t)$ must also be a Gaussian random process.

In order to determine the laser spectrum, first we need to know the autocorrelation function $\Phi_{E E}(\tau)$ of the signal $E(t)$, which can be defined as

$$
\begin{array}{r}
\Phi_{E E}(\tau)=E\{E(t+\tau) E(t)\}=\operatorname{Re}\left\{\Phi_{E_{l} E_{l}}(\tau) e^{j 2 \pi f_{0} \tau}\right\}, \\
-\infty<\tau<\infty,
\end{array}
$$

where

$$
\Phi_{E_{l} E_{l}}(\tau)=\frac{1}{2} E\left\{E_{l}(t+\tau) E_{l}^{*}(t)\right\}
$$

is the autocorrelation function of the complex envelope $E_{l}(t)$ of signal $E(t), E\{\cdot\}$ is the operator of ensemble averaging (expected value), and $*$ represents complex conjugation. The power spectrum $\Phi_{E E}(f)$ of unmodulated laser signal $E(t)$ can be determined by using the autocorrelation theorem. From this theorem, it is clear that double-sided power spectral density $\Phi_{E E}(f)$ of signal $E(t)$ is represented by the Fourier transform of the autocorrelation function $\Phi_{E E}(\tau)$ of the signal $E(t)[23]$ and then

$$
\begin{array}{r}
\Phi_{E E}(f)=\mathbb{F}\left\{\Phi_{E E}(\tau)\right\}(f)=\int_{-\infty}^{\infty} \Phi_{E E}(\tau) e^{-j 2 \pi f \tau} d \tau, \\
-\infty<f<\infty
\end{array}
$$

where $\mathbb{F}\{\cdot\}$ denotes Fourier transform. From a physical point of view, we take into account only those spectral components that are situated on the positive frequencies $f$. Instead of double-sided PSD, we use only single-sided PSD of unmodulated signal, here denoted as $\widehat{\Phi}_{E E}(f)$. Single-sided PSD is defined as follows:

$$
\widehat{\Phi}_{E E}(f)= \begin{cases}2 \Phi_{E E}(f), & 0 \leq f<\infty, \\ 0, & -\infty<f<0 .\end{cases}
$$

It is suitable to operate with shifted version of single-sided $\operatorname{PSD} \widehat{\Phi}_{E E}(f)$ of unmodulated laser signal $E(t)$. The frequency shift toward zero frequency by frequency value $f_{0}$ is performed. Therefore, we obtain the power spectrum centred around zero frequency. It can be shown that this operation is valid and it is described in more detail in Appendix A. As a result of this approach, we get

$$
\widehat{\Phi}_{E E}\left(f+f_{0}\right)=\mathbb{F}\left\{\Phi_{E_{l} E_{l}}(\tau)\right\}(f), \quad-\infty<f<\infty .
$$

In the following, we will determine the unmodulated signal spectrum only by using (9). Therefore as a first step, it is suitable to determine the autocorrelation function of complex envelope of unmodulated laser signal. By substituting (3) into (6), we get

$$
\Phi_{E_{l} E_{l}}(\tau)=P_{0} E\left\{e^{j\left[\theta_{E}(t+\tau)-\theta_{E}(t)\right]}\right\}
$$

For Gaussian random variable, the following relation is valid $[23,24]$ :

$$
E\left\{e^{j X}\right\}=e^{-(1 / 2) E\left\{X^{2}\right\}}
$$

If we utilize (11), (10) becomes of the form

$$
\Phi_{E_{l} E_{l}}(\tau)=P_{0} e^{-(1 / 2) E\left\{\left[\theta_{E}(t+\tau)-\theta_{E}(t)\right]^{2}\right\}} .
$$

Based on (4), for the difference of the instantaneous phase values at the two different time instants, which are $\tau$ seconds apart from each other on the time scale $t$, we can write down the following expression:

$$
\theta_{E}(t+\tau)-\theta_{E}(t)=2 \pi \int_{t}^{t+\tau} \nu(\alpha) d \alpha .
$$

The statistical averaging of square of (13) is valid (see Appendix B)

$$
\begin{aligned}
E\{ & {\left.\left[\theta_{E}(t+\tau)-\theta_{E}(t)\right]^{2}\right\} } \\
& =4 \pi^{2} \int_{-|\tau|}^{|\tau|}(|\tau|-|u|) \Phi_{\nu v}(u) d u
\end{aligned}
$$

where $\Phi_{\nu \nu}(u)$ is the autocorrelation function of the phase noise $v(t)$ and it is valid that

$$
\Phi_{\nu v}(u)=E\{v(t+u) v(t)\}
$$

Equation (14) can be adjusted to the form

$$
\begin{aligned}
E\{ & {\left.\left[\theta_{E}(t+\tau)-\theta_{E}(t)\right]^{2}\right\} } \\
& =4 \pi^{2} \int_{-\infty}^{\infty}|\tau| \operatorname{tri}\left(\frac{u}{2|\tau|}\right) \Phi_{\nu \nu}(u) d u,
\end{aligned}
$$

where the triangular function $\operatorname{tri}(x)$ is expressed as follows:

$$
\operatorname{tri}(x)=\left\{\begin{array}{lc}
1-2|x|, & -\frac{1}{2}<x<\frac{1}{2}, \\
0, & \text { otherwise. }
\end{array}\right.
$$

From the autocorrelation theorem, we can obtain the autocorrelation function $\Phi_{\nu v}(u)$ of the phase noise $v(t)$ by using the inverse Fourier transform of double-sided $\operatorname{PSD} \Phi_{\nu v}(f)$ of the phase noise

$$
\Phi_{\nu v}(u)=\mathbb{F}^{-1}\left\{\Phi_{\nu v}(f)\right\}(u)=\int_{-\infty}^{\infty} \Phi_{\nu v}(f) e^{j 2 \pi f u} d f .
$$


By substituting for $\Phi_{\nu v}(u)$ from (18) to (16), we get

$$
\begin{gathered}
E\left\{\left[\theta_{E}(t+\tau)-\theta_{E}(t)\right]^{2}\right\}=4 \pi^{2} \int_{-\infty}^{\infty}|\tau| \operatorname{tri}\left(\frac{u}{2|\tau|}\right) \\
\cdot\left[\int_{-\infty}^{\infty} \Phi_{\nu v}(f) e^{j 2 \pi f u} d f\right] d u .
\end{gathered}
$$

After the change of the integration order and adjusting the previous equation, we get

$$
\begin{aligned}
& E\left\{\left[\theta_{E}(t+\tau)-\theta_{E}(t)\right]^{2}\right\}=4 \pi^{2}|\tau| \\
& \cdot \int_{-\infty}^{\infty} \Phi_{\nu \nu}(f)\left[\int_{-\infty}^{\infty} \operatorname{tri}\left(\frac{u}{2|\tau|}\right) e^{j 2 \pi f u} d u\right] d f .
\end{aligned}
$$

For the expression in the square brackets on the right side in the last equation, the following is valid:

$$
\begin{aligned}
& \int_{-\infty}^{\infty} \operatorname{tri}\left(\frac{u}{2|\tau|}\right) e^{j 2 \pi f u} d u=\left[\mathbb{F}\left\{\operatorname{tri}\left(\frac{u}{2|\tau|}\right)\right\}(f)\right]^{*} \\
& =|\tau|[\operatorname{sinc}(|\tau| f)]^{2},
\end{aligned}
$$

where the function $\operatorname{sinc}(x)$ is defined as

$$
\operatorname{sinc}(x)= \begin{cases}1, & x=0, \\ \frac{\sin (\pi x)}{\pi x}, & x \neq 0 .\end{cases}
$$

By substituting (21) into (19), the mean square value of difference of the instantaneous phase $\theta_{E}(t)$ at the two different time instants which are $\tau$ seconds apart from each other on the time scale $t$ is

$$
\begin{aligned}
E\{ & {\left.\left[\theta_{E}(t+\tau)-\theta_{E}(t)\right]^{2}\right\} } \\
& =4 \pi^{2} \tau^{2} \int_{0}^{\infty} \widehat{\Phi}_{\nu \nu}(f)[\operatorname{sinc}(|\tau| f)]^{2} d f
\end{aligned}
$$

where $\widehat{\Phi}_{v v}(f)$ is single-sided PSD of the phase noise $v(t)$, which is defined as follows:

$$
\widehat{\Phi}_{\nu v}(f)= \begin{cases}2 \Phi_{\nu \nu}(f), & 0 \leq f<\infty, \\ 0, & -\infty<f<0 .\end{cases}
$$

It is suitable to introduce another parameter which is indirectly describing the spectral properties of the unmodulated signal of the laser $E(t)$. This parameter is average power $P_{\nu}$ of the phase noise $v(t)$ which is given by following relation:

$$
P_{\nu}=\int_{0}^{\infty} \widehat{\Phi}_{\nu v}(f) d f=\int_{-\infty}^{\infty} \Phi_{\nu v}(f) d f .
$$

At the beginning of this section, we assumed that the phase noise $\nu(t)$ is the stationary Gaussian random process and in the baseband of bandwidth $B_{v}$, the phase noise $v(t)$ has a white noise character. From this reason, it is possible to express the single-sided PSD $\widehat{\Phi}_{\nu v}(f)$ as follows:

$$
\widehat{\Phi}_{v v}(f)= \begin{cases}\frac{P_{v}}{B_{v}}, & 0 \leq f \leq B_{v}, \\ 0, & B_{v}<f<\infty .\end{cases}
$$

After substitution of $\widehat{\Phi}_{\nu v}(f)$ from (26) to (23) and after further adjustment, we get

$$
\begin{aligned}
& E\left\{\left[\theta_{E}(t+\tau)-\theta_{E}(t)\right]^{2}\right\} \\
& \quad=4 \pi|\tau| \frac{P_{v}}{B_{v}} \int_{0}^{\pi B_{v}|\tau|}\left[\frac{\sin (x)}{x}\right]^{2} d x .
\end{aligned}
$$

The unmodulated laser signal can be viewed as one spectral line located at the frequency $f_{0}$ with the zero bandwidth that is frequency modulated (FM) by phase noise and causing spectral line spreading. Thus, the real lasers have nonzero bandwidth, which is caused exactly by this frequency modulation. Usually the bandwidth is characterized by full width at half maximum, which is denoted as $\Delta_{\text {FWHM }}$. The frequency deviation of that FM is equal to the average value of the instantaneous frequency deviation of the signal $E(t)$ from frequency $f_{0}[14,17,18,20]$. Instantaneous value of this deviation is denoted as $\nu(t)$ and its average value is equal to $\sqrt{P_{v}}$. Afterwards, the quantity $\sqrt{P_{v}}$ is considered as a frequency deviation of the aforementioned frequency modulation. Because the bandwidth of modulating signal $v(t)$ is $B_{v}$, then the index of FM modulation is $\sqrt{P_{v}} / B_{v}$. The quantity of phase noise $v(t)$ has dimensions in $\mathrm{Hz}$, and its average power $P_{v}$ is the quantity expressed in $\mathrm{Hz}^{2}$; then the expression $\sqrt{P_{v}} / B_{v}$ is dimensionless. As we will show below, the value $\sqrt{P_{v}} / B_{v}$ will determine the character of the unmodulated laser spectrum $[17,20]$.

The value of the FM modulation index determines two characteristics of power spectra of the unmodulated laser signal. For a case, where the index of FM modulation is small, that is, $B_{v}$ is large enough compared to $\sqrt{P_{v}}$, the resulting spectrum shape is Lorentzian. For a case, when the FM index is high, that is, $B_{v}$ is small enough compared to $\sqrt{P_{v}}$, the final spectrum follows the Gaussian shape.

In the first case, where $B_{v}$ is large compared to $\sqrt{P_{v}}$, that is, $B_{\nu}$ increases to the infinity, for the integral on the right side of (27), we can write this approximation

$$
\int_{0}^{\pi B_{y}|\tau|}\left[\frac{\sin (x)}{x}\right]^{2} d x \approx \frac{\pi}{2}
$$

and (27) becomes

$$
E\left\{\left[\theta_{E}(t+\tau)-\theta_{E}(t)\right]^{2}\right\} \approx 2 \pi^{2} \frac{P_{v}}{B_{v}}|\tau|
$$

By substituting the last expression to (12), then for the autocorrelation function of the complex envelope of the unmodulated laser signal, the following is valid:

$$
\Phi_{E_{l} E_{l}}(\tau)=P_{0} e^{-\pi^{2}\left(P_{\nu} / B_{\nu}\right)|\tau|}, \quad-\infty<\tau<\infty .
$$

By using (9), we can obtain the power spectrum of the unmodulated laser signal shifted to the zero frequency region. This step is performed by using Fourier transform of the 
autocorrelation function of complex envelope. Then the power spectrum can be expressed as follows:

$$
\begin{aligned}
& \widehat{\Phi}_{E E}\left(f+f_{0}\right)=\frac{P_{0}}{\pi} \frac{(2 / \pi)\left(B_{v} / P_{\nu}\right)}{1+\left((2 / \pi)\left(B_{\nu} / P_{\nu}\right) f\right)^{2}} \\
&-\infty<f<\infty .
\end{aligned}
$$

The shape of the power spectral density follows Lorentzian shape and the FWHM is given by

$$
\Delta_{\mathrm{FWHM}}=\pi \frac{P_{v}}{B_{v}} .
$$

Assuming that the root mean square (RMS) value $\sqrt{P_{v}}$ of the phase noise $\nu(t)$ is much lower than the bandwidth $B_{v}$, then the linewidth of the unmodulated laser signal definitely depends on the power spectral density of the phase noise. In the second case, where $B_{v}$ is small compared to $\sqrt{P_{v}}$, the integral on the right side of (27) is rewritten as follows:

$$
\int_{0}^{\pi B_{v}|\tau|}\left[\frac{\sin (x)}{x}\right]^{2} d x \approx \pi B_{v}|\tau|
$$

and (27) becomes to

$$
E\left\{\left[\theta_{E}(t+\tau)-\theta_{E}(t)\right]^{2}\right\} \approx 4 \pi^{2} P_{\nu} \tau^{2} .
$$

By substituting the last expression to (12), the autocorrelation function of the complex envelope of the unmodulated laser signal can be expressed as

$$
\Phi_{E_{l} E_{l}}(\tau)=P_{0} e^{-2 \pi^{2} P_{\nu} \tau^{2}}, \quad-\infty<\tau<\infty .
$$

By using (9), we can apply the Fourier transform on the last equation. This way, we obtain the power spectrum of the unmodulated laser signal shifted to the zero frequency as

$$
\widehat{\Phi}_{E E}\left(f+f_{0}\right)=\frac{P_{0}}{\sqrt{2 \pi P_{v}}} e^{-f^{2} / 2 P_{\nu}}, \quad-\infty<f<\infty .
$$

From the last expression it is obvious that the power spectral density has a Gaussian shape and the FWHM can be expressed as follows:

$$
\Delta_{\mathrm{FWHM}}=2 \sqrt{2 \ln (2)} \sqrt{P_{v}}
$$

By considering the condition that the RMS of the phase noise $\sqrt{P_{v}}$ is much larger than the bandwidth $B_{v}$, then the linewidth of unmodulated laser signal definitely depends on the RMS of phase noise $\sqrt{P_{v}}$.

\section{Numerical Simulation and Results}

In this section, we present the application of the aforedescribed theoretical analysis to estimate the linewidth of distributed feedback semiconductor laser (DFB) by using the numerical approach. The DFB laser model is based on the standard equivalent circuit model $[21,22]$. Here, the model is built up by using a set of coupled differential equations, where the noise is modelled via Langevin noise sources. The light emitting sources analysed by this approach have output characteristics that are comparable to the demonstrated experiments [21, 22]. The main output parameters of the numerical model are time-dependent optical power $p(t)$ and its instantaneous phase $\theta_{E}(t)$. The signal characteristics of the numerical model are described in $[9,21]$.

The time-dependent optical power $p(t)$ and the frequency fluctuations $\nu(t)$ were generated for following parameters. Specifically, we considered two wavelengths of $1310 \mathrm{~nm}$ and $1550 \mathrm{~nm}$, respectively, different input powers of the laser, ranging from $12.5 \mathrm{~mA}$ to $25 \mathrm{~mA}$, and various levels of Langevin noise sources. In the case that Langevin noise sources satisfy Markovian assumptions [22], the noise sources are appropriately simulated by three random Gaussian variables with zero mean value and variance $\sigma$. We used the parameter $\sigma$ to modify the impact of noise in the numerical model. Particular time-dependent variables were generated in the time interval $T=400 \mathrm{~ns}$ with integration (time) step $\Delta t=10 \mathrm{ps}$. This integration step is sufficient to achieve the numerical stability of fourth-order Runge-Kutta method that was used to solve the laser rate equations $[10,21,22$, 25]. From the statistical point of view, we performed $N=$ 3000 realizations for the time-dependent optical powers $p(t)$ series and fluctuations of the instantaneous frequency $\nu(t)$ that deviates from the reference frequency $f_{0}$. From the generated time-dependent instantaneous powers $p(t)$, we have determined the estimation of $P_{0}$ as

$$
P_{0}=\frac{1}{N} \sum_{n=1}^{N} \frac{1}{T} \int_{0}^{T} p_{n}(t) d t
$$

where $N$ is the number of time-dependent realizations, index $n$ is the number of the particular realisation of $p(t)$ and $\nu(t)$, and $T$ is the duration of these realizations. Subsequently, we have determined the one-sided power spectral density (see Figure 1) of the phase noise $\widehat{\Phi}_{\nu \nu}(f)$ as follows:

$$
\widehat{\Phi}_{\nu v}(f)=\frac{2}{T}|\mathbb{F}\{\nu(t)\}(f)|^{2}
$$

$\widehat{\Phi}_{\nu \nu}(f)$ is determined only for positive frequencies from each realization of $v(t)$. Then, the values of the individual power spectral densities (particular statistical realisation) were averaged. As a result, we obtained a one mean singlesided power spectral density of the phase noise.

It is worth noting that, at the beginning of each individual realization, the laser transient state comes into play. This artificial effect has to be suppressed in order to correctly estimate the power spectrum. The suppression of the undesired laser transient states can be performed in the frequency domain as follows:

$$
\widehat{\Phi}_{\nu v}(f)=\widehat{\widehat{\Phi}}_{\nu v}(f)-\frac{2}{T}\left|\mathbb{F}\left\{\mu_{v}(t)\right\}(f)\right|^{2},
$$




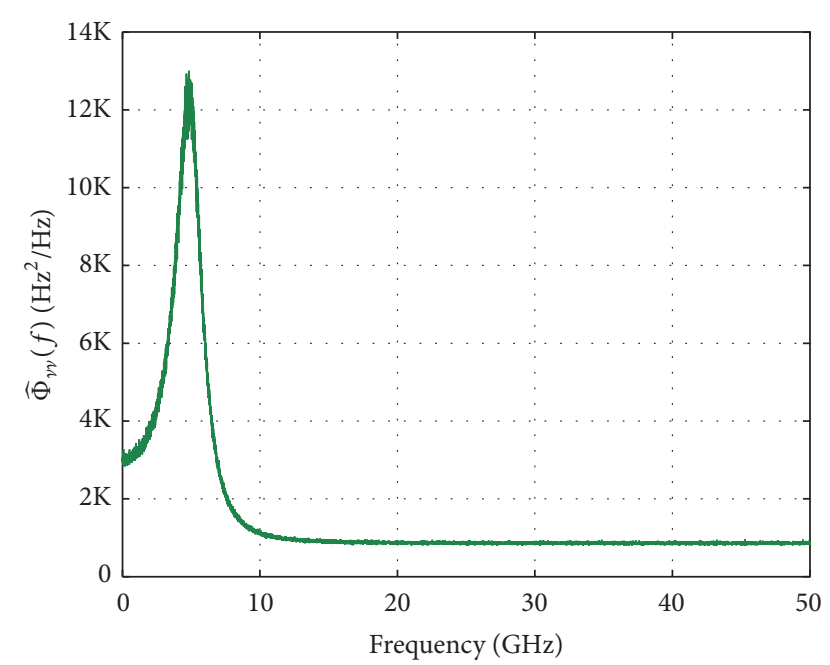

FIGURE 1: Averaged one-sided power spectral density of $\nu(t)$ for $\lambda=$ $1310 \mathrm{~nm}, I_{\text {in }}=25 \mathrm{~mA}$, and $\sigma=0.1$.

where $\bar{\Phi}_{v v}(f)$ is the estimation of one-sided power spectral density of phase noise affected by transient process and the average time-dependent transient process

$$
\mu_{\nu}(t)=\frac{1}{N} \sum_{n=1}^{N} v_{n}(t)
$$

is obtained by time averaging of all realisations $\nu(t)$.

By using (23), the root mean square value of difference of the two values of instantaneous phase $\theta_{E}(t)$ at the two different time instants which are $\tau$ seconds apart from each other on the time scale $t$ was calculated. Subsequently, by substituting this result into (12), we obtained the autocorrelation function of the complex envelope of the laser signal.

To determine the power spectrum $\widehat{\Phi}_{E E}\left(f+f_{0}\right)$ of the unmodulated laser signal $E(t)$ from autocorrelation function $\Phi_{E_{1} E_{l}}(\tau)$ according to (9), we could use cosine transform instead of Fourier transform. This approach is valid, because the autocorrelation function $\Phi_{E_{l} E_{l}}(\tau)$ is an even function (see Figure 2).

From the reason of higher numerical accuracy, only those samples of power spectrum $\widehat{\Phi}_{E E}\left(f+f_{0}\right)$ are computed by cosine transform that matches the frequency grid of the original signal $E(t)$ in the spectral domain. Subsequently, those samples are approximated by function that describes Voigt probability function. This function is the linear combination of Gaussian and Lorentzian probability function [26].

In Figure 3 the approximation of the computed spectrum samples by Voigt profile at a wavelength of $1310 \mathrm{~nm}$ is depicted. Here, the input current of laser was set to $25 \mathrm{~mA}$ and the value of parameter $\sigma=0.1$. It is clear that output power spectrum is given not only by the value of FM modulation (small or large), that is, Lorentzian or Gaussian profile, but by their combination. As can be seen below, the theoretical laser linewidth will be in the range given by expressions (32) and (37). For example, the estimated value of the laser linewidth at the wavelength $\lambda_{0}=1310 \mathrm{~nm}$ and input power $I_{\text {in }}=25 \mathrm{~mA}$

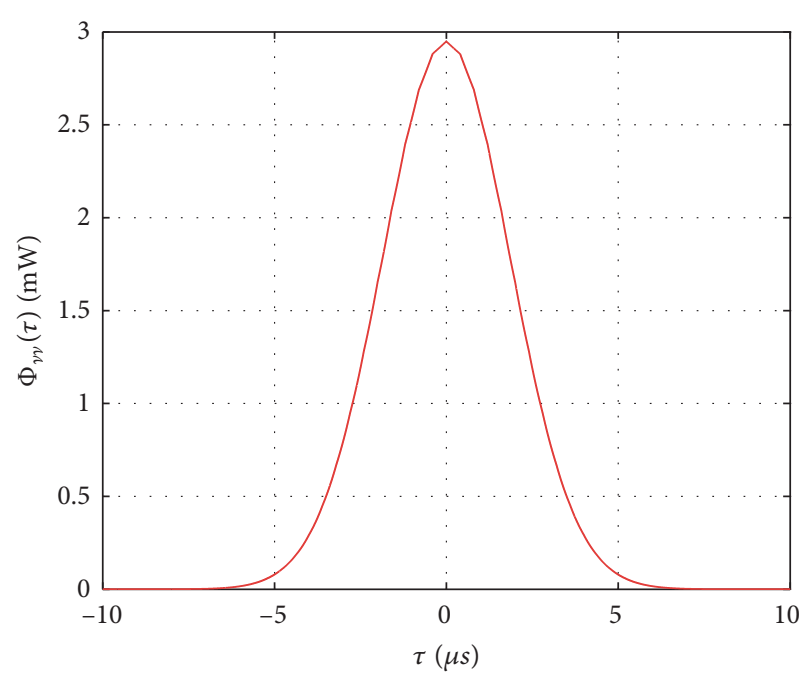

FIgURE 2: Autocorrelation function of the complex envelope of the unmodulated laser signal.

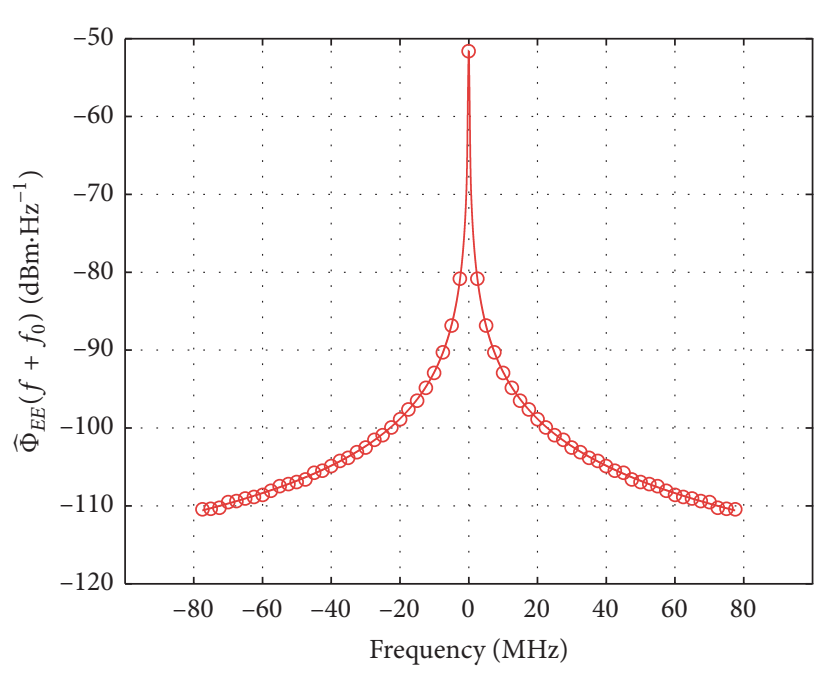

FIGURE 3: Calculated samples and approximation of power spectrum $\widehat{\Phi}_{E E}\left(f+f_{0}\right)$ by Voigt profile.

and for the parameter $\sigma=0.1$ was $0.2526 \mathrm{MHz}$, while the calculated value by (32) was $21.3264 \mathrm{MHz}$ and by (37) was $0.0052 \mathrm{MHz}$. These two values are only a rough estimate. The exact value is obtained through simulation.

In Figure 4 the dependence of output average laser power as a function of input current $I_{\text {in }}$ is shown at the wavelengths of $1550 \mathrm{~nm}$ and $1310 \mathrm{~nm}$, respectively. These wavelengths are attractive for currently used optical communication systems. The threshold level of the input current is $10.2 \mathrm{~mA}[9,21]$. Threshold level of the injected current to the laser determines the lowest excitation level, at which laser operates in the regime of stimulated emission. From this reason the injected currents above the threshold are used.

In Figures 5 and 6, the dependence of laser linewidth for various values of the parameter $\sigma$ is shown. Simulation shows that results of the numerical model are in the range from few 


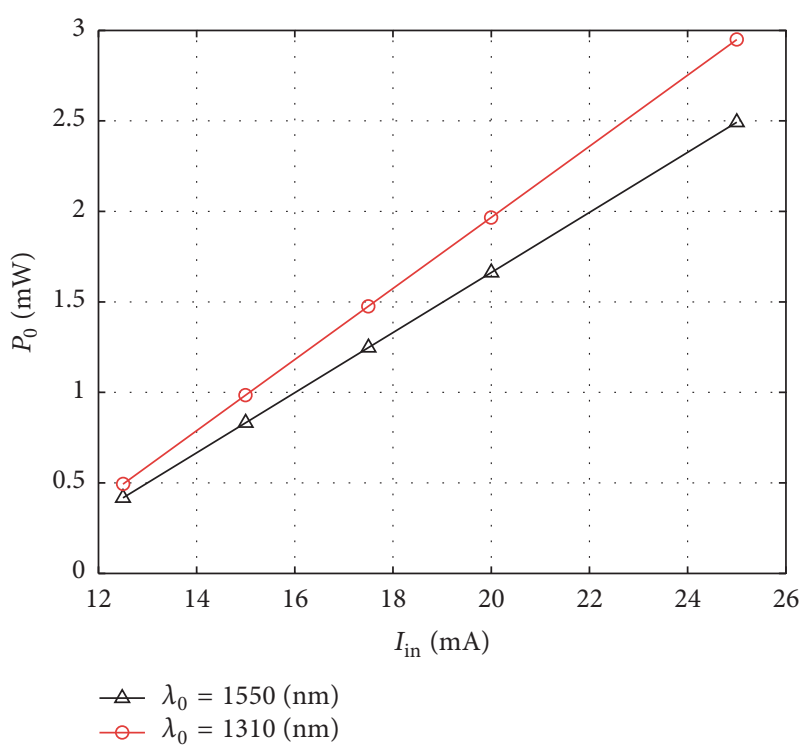

FIGURE 4: Dependence of average output laser power at wavelengths $1550 \mathrm{~nm}$ and $1310 \mathrm{~nm}$ for different values of input current.

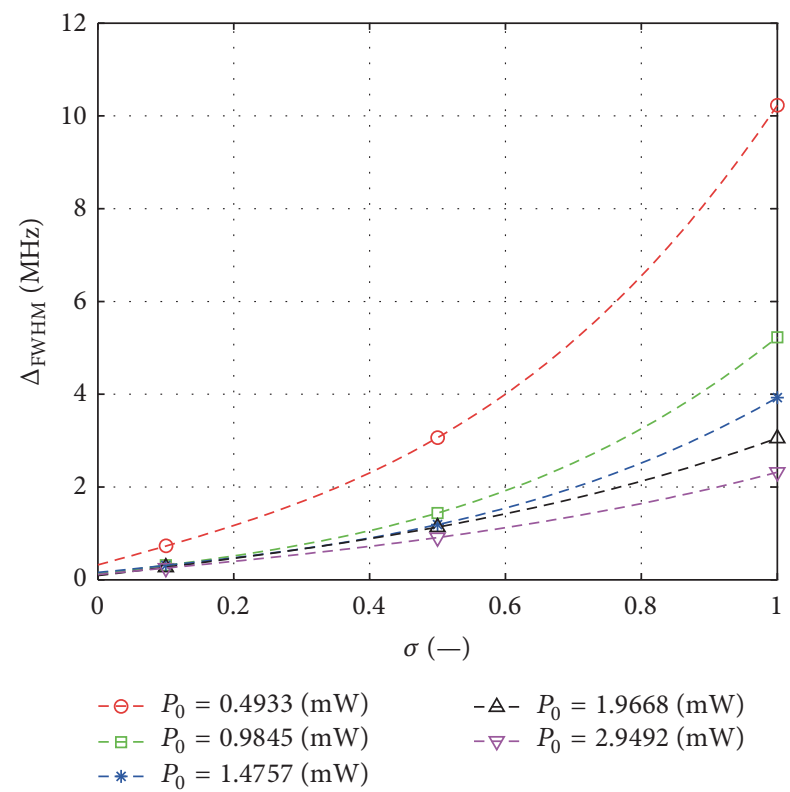

FIGURE 5: Approximated values of semiconductor DFB laser linewidth obtained by numerical approach for wavelength $1310 \mathrm{~nm}$.

$\mathrm{kHz}$ to few tens of MHz. The laser with the narrowest spectral linewidth is characterized by the highest output average power $P_{0}$ and with the smallest value of $\sigma$. Concretely for the following parameters $I_{\text {in }}=25 \mathrm{~mA}$ and $P_{0}=2.9492 \mathrm{~mW}$ and $\sigma=0.1$ is on the wavelength $1310 \mathrm{~nm}$, the value of the spectral linewidth of laser $252.6 \mathrm{kHz}$.

On the other hand, the laser with the smallest output power $P_{0}=0.4169 \mathrm{~mW}$ and $\sigma=1$ on the wavelength $1550 \mathrm{~nm}$ has linewidth $10.786 \mathrm{MHz}$. The difference of spectral linewidth values for particular wavelength is given by the average output power $P_{0}$, which is different for different

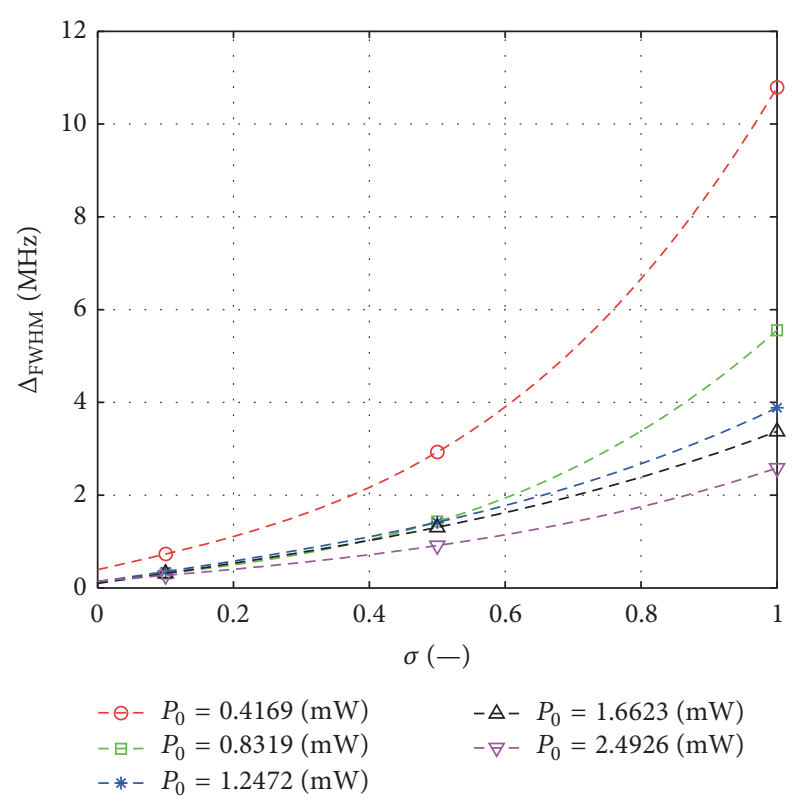

FIGURE 6: Approximated values of semiconductor DFB laser linewidth obtained by numerical approach for wavelength $1550 \mathrm{~nm}$.

wavelengths. From this reason the laser emitting at the wavelength of $1310 \mathrm{~nm}$ achieves slightly better values of laser linewidth compared to the wavelength $1550 \mathrm{~nm}$.

\section{Conclusion}

In summary, we reported on the detailed mathematical methodology, supported by numerical calculations based on laser rate equations, to accurately estimate the laser spectral properties. In particular, we developed a straightforward theoretical approach to numerically evaluate the spectral characteristics of optical sources, with the focus on distributed feedback semiconductor lasers, typically used by fiber-optic communications systems. This description and presented numerical model are appropriate in situations, when time-dependent laser parameters are readily attainable, specifically output laser power and its instantaneous optical phase. Here, the mathematical analysis provided in the work directly begins with the complex envelope of the laser signal in the baseband and this approach is advantageously used to characterize desired parameters of optical sources, especially laser linewidth, as numerically demonstrated and evaluated in this work. Furthermore, this work provides explicit characterization of the laser linewidth in the baseband, being a parameter of practical importance, because it can be shown by the optical spectral analysers. Specifically, the laser power spectral density was simulated using two important near-infrared wavelengths of $1310 \mathrm{~nm}$ and $1550 \mathrm{~nm}$. This was done for various injection currents and different levels of the Langevin noise sources. The narrowest laser linewidth obtained by simulation was $252.6 \mathrm{kHz}$. This value was achieved for the highest average laser output power of $2.9492 \mathrm{~mW}$ and for the lowest parameter $\sigma=0.1$. On the other hand, the largest laser linewidth of $10.768 \mathrm{MHz}$ was 
reached on the lowest average power for the highest value of the parameter $\sigma=1$. The methodology we presented in this work affords compact mathematical form, clearly described and well-connected to the terms and concepts of modern theory of optical digital communications. In addition, the reported theoretical method for estimation of laser spectral properties can be potentially used to optimize laser parameters in order to extend the examination of its noise properties, which, in turn, can be beneficially utilized for further numerical investigation of the impact of laser noise in recent large wavelength-channel count optical transmission systems, utilizing the multilevel modulation techniques and principles of coherent detection.

\section{Appendix}

\section{A. Formula for Single-Sided Power Spectral Density}

In this part, the shifted power spectrum to the zero frequency area, which is expressed by (9), is derived. We started from (5), which express autocorrelation function of $\Phi_{E E}(\tau)$. At the first step, relationship for evaluation of the real part of complex number is applied

$$
\operatorname{Re}\{z\}=\frac{1}{2} z+\frac{1}{2} z^{*}
$$

on (5). The (5) goes to form

$$
\Phi_{E E}(\tau)=\frac{1}{2} \Phi_{E_{l} E_{l}}(\tau) e^{j 2 \pi f_{0} \tau}+\frac{1}{2} \Phi_{E_{l} E_{l}}^{*}(\tau) e^{-j 2 \pi f_{0} \tau} .
$$

On the base of autocorrelation theorem, which is used in (7) and previous equation, the double-sided $\operatorname{PSD} \Phi_{E E}(f)$ is given as

$$
\begin{aligned}
\Phi_{E E}(f)= & \frac{1}{2} \mathbb{F}\left\{\Phi_{E_{l} E_{l}}(\tau)\right\}\left(f-f_{0}\right) \\
& +\frac{1}{2} \mathbb{F}\left\{\Phi_{E_{l} E_{l}}^{*}(\tau)\right\}\left(f+f_{0}\right) .
\end{aligned}
$$

The single-sided PSD $\widehat{\Phi}_{E E}(f)$ is derived from double-sided $\operatorname{PSD} \Phi_{E E}(f)$ by using Heaviside step function $u(f)$ as follows:

$$
\widehat{\Phi}_{E E}(f)=2 u(f) \Phi_{E E}(f), \quad-\infty<f<\infty .
$$

By substitution (A.3) to (A.4), then single-sided PSD goes to the form

$$
\begin{aligned}
\widehat{\Phi}_{E E}(f)= & 2 u(f)\left[\frac{1}{2} \mathbb{F}\left\{\Phi_{E_{l} E_{l}}(\tau)\right\}\left(f-f_{0}\right)\right. \\
& \left.+\frac{1}{2} \mathbb{F}\left\{\Phi_{E_{l} E_{l}}(-\tau)\right\}\left(f+f_{0}\right)\right] .
\end{aligned}
$$

From the properties of Heaviside step function the last term on the right side of (A.5) is equal to zero. By substituting $f \rightarrow$ $f+f_{0}$ to (A.5) we get single-sided PSD

$$
\widehat{\Phi}_{E E}\left(f+f_{0}\right)=\mathbb{F}\left\{\Phi_{E_{l} E_{l}}(\tau)\right\}(f) .
$$

\section{B. Expression of Statistical Averaging for Squared Difference of Instantaneous Phase}

In this part of the appendix the statistical averaging of squared difference of instantaneous phase values which are expressed in (14) is derived. We have to divide the whole interval of values of the time parameter $\tau$ to the two subintervals: the first one for positive values and the second one for negative values, respectively. So the time parameter $\tau$ is divided into the negative and positive valued regions. According to (4) and (13) the difference of the instantaneous phase at the two different time instants which are $\tau$ seconds apart from each other on the time scale $t$ is as follows:

(1) For $-\infty<\tau<0$

$$
\begin{aligned}
\theta_{E}(t+\tau)-\theta_{E}(t) & =\theta_{E}(t-|\tau|)-\theta_{E}(t) \\
& =-2 \pi \int_{t-|\tau|}^{t} \nu(\alpha) d \alpha .
\end{aligned}
$$

(2) For $0 \leq \tau<\infty$

$$
\begin{aligned}
\theta_{E}(t+\tau)-\theta_{E}(t) & =\theta_{E}(t+|\tau|)-\theta_{E}(t) \\
& =2 \pi \int_{t}^{t+|\tau|} \nu(\alpha) d \alpha .
\end{aligned}
$$

By squaring of relationships (B.1) and (B.2), we get

(1) for $-\infty<\tau<0$

$$
\begin{aligned}
{\left[\theta_{E}(t+\tau)-\theta_{E}(t)\right]^{2} } & =\left[-2 \pi \int_{t-|\tau|}^{t} \nu(\alpha) d \alpha\right]^{2} \\
& =4 \pi^{2} \iint_{t-|\tau|}^{t} \nu(\alpha) \nu(\beta) d \alpha d \beta,
\end{aligned}
$$

(2) for $0 \leq \tau<\infty$

$$
\begin{aligned}
{\left[\theta_{E}(t+\tau)-\theta_{E}(t)\right]^{2} } & =\left[2 \pi \int_{t}^{t+|\tau|} \nu(\alpha) d \alpha\right]^{2} \\
& =4 \pi^{2} \iint_{t}^{t+|\tau|} \nu(\alpha) \nu(\beta) d \alpha d \beta .
\end{aligned}
$$

Applying the ensemble averaging operator $E\{\cdot\}$ to the last two equations, we get

(1) for $-\infty<\tau<0$

$$
\begin{aligned}
E\{ & {\left.\left[\theta_{E}(t+\tau)-\theta_{E}(t)\right]^{2}\right\} } \\
& =4 \pi^{2} \iint_{t-|\tau|}^{t} E\{\nu(\alpha) \nu(\beta)\} d \alpha d \beta,
\end{aligned}
$$

(2) for $0 \leq \tau<\infty$

$$
\begin{aligned}
E\{ & {\left.\left[\theta_{E}(t+\tau)-\theta_{E}(t)\right]^{2}\right\} } \\
& =4 \pi^{2} \iint_{t}^{t+|\tau|} E\{\nu(\alpha) \nu(\beta)\} d \alpha d \beta .
\end{aligned}
$$


Let us denote $E\{\nu(\alpha) \nu(\beta)\}$ as $\Phi_{\nu v}(\alpha, \beta)$, the autocorrelation function of $\nu(t)$, the function of instantaneous frequency fluctuations of the unmodulated signal of the laser from its mean frequency. On the base of the condition of stationarity (see assumptions in Section 2) it is possible to express $\Phi_{\nu v}(\alpha, \beta)$ as $\Phi_{\nu v}(\alpha-\beta)$. Then relations (B.5) and (B.6) go to

(1) for $-\infty<\tau<0$

$$
\begin{aligned}
E\{ & {\left.\left[\theta_{E}(t+\tau)-\theta_{E}(t)\right]^{2}\right\} } \\
& =4 \pi^{2} \iint_{t-|\tau|}^{t} \Phi_{\nu \nu}(\alpha-\beta) d \alpha d \beta,
\end{aligned}
$$

(2) for $0 \leq \tau<\infty$

$$
\begin{aligned}
E\{ & {\left.\left[\theta_{E}(t+\tau)-\theta_{E}(t)\right]^{2}\right\} } \\
& =4 \pi^{2} \iint_{t}^{t+|\tau|} \Phi_{\nu v}(\alpha-\beta) d \alpha d \beta .
\end{aligned}
$$

For simplification, the coordinate transformation by changing of variables in the double integral in (B.7) and (B.8) is applied [27]. In the first step the area $O(\alpha, \beta)$ is defined:

(1) For $-\infty<\tau<0$ the area $O(\alpha, \beta)$ is defined as follows: for this range of $\beta: t-|\tau| \leq \beta \leq t$, the range $t-|\tau| \leq \alpha \leq t$ for $\alpha$ is defined.

(2) For $0 \leq \tau<\infty$ the area $O(\alpha, \beta)$ is defined as follows: for this range of $\beta: t \leq \beta \leq t+|\tau|$,

the range $t \leq \alpha \leq t+|\tau|$ for $\alpha$ is defined.

In both cases this transformation of coordinates $\alpha, \beta$ is applied:

$$
\begin{aligned}
& u=\alpha-\beta, \\
& v=\alpha+\beta .
\end{aligned}
$$

After the coordinate system transformation the area $O(\alpha, \beta)$ goes to the area $O(u, v)$ as follows:

(1) For $-\infty<\tau<0$ the area $O(u, v)$ is expressed as follows:

(a) For this range of $u$ : $-|\tau| \leq u \leq 0$, the range $v_{1} \leq v \leq v_{2}$ of $v$ is defined, where for boundary values $v_{1}$ and $v_{2}$ these statements hold:

$$
\begin{aligned}
& v_{1}=2(t-|\tau|)-u, \\
& v_{2}=2 t+u .
\end{aligned}
$$

(b) For this range of $u$ : $0 \leq u \leq|\tau|$, the range $v_{3} \leq v \leq v_{4}$ of $v$ is defined, where for boundary values $v_{3}$ and $v_{4}$ these statements hold:

$$
\begin{aligned}
& v_{3}=2(t-|\tau|)+u, \\
& v_{4}=2 t-u .
\end{aligned}
$$

(2) For $0 \leq \tau<\infty O(u, v)$ is expressed as follows:

(a) For this range of $u$ : $-|\tau| \leq u \leq 0$, the range $v_{5} \leq v \leq v_{6}$ of $v$ is defined, where for boundary values $v_{5}$ and $v_{6}$ these statements hold:

$$
\begin{aligned}
& v_{5}=2 t-u \\
& v_{6}=2(t+|\tau|)+u
\end{aligned}
$$

(b) For this range of $u$ : $0 \leq u \leq|\tau|$, the range $v_{7} \leq v \leq v_{8}$ of $v$ is defined, where for boundary values $v_{7}$ and $v_{8}$ these statements hold:

$$
\begin{aligned}
& v_{7}=2 t+u \\
& v_{8}=2(t+|\tau|)-u
\end{aligned}
$$

From (B.12) we are able to define the inverse transformation equations of coordinates $u, v$ to the coordinates $\alpha, \beta$. This transformation is defined through functions $x(u, v)$ and $y(u, v)$ as follows:

$$
\begin{aligned}
& \alpha=x(u, v)=\frac{v+u}{2}, \\
& \beta=y(u, v)=\frac{v-u}{2} .
\end{aligned}
$$

From (B.17) we are able to define $d \alpha d \beta$ which appears in (B.7) and (B.8) as follows:

$$
d \alpha d \beta=R d v d u
$$

where $R$ is the Jacobian for which the following holds:

$$
\begin{aligned}
R & =\frac{\partial}{\partial u} x(u, v) \frac{\partial}{\partial v} y(u, v)+-\frac{\partial}{\partial u} y(u, v) \frac{\partial}{\partial v} x(u, v) \\
& =\frac{1}{2}
\end{aligned}
$$

On the base of the abovementioned, it is possible to express the double integrals in (B.7) and (B.8) as follows:

(1) For $-\infty<\tau<0$ and by using (B.9), (B.13), (B.14), and (B.18) for double integral in (B.7), we obtain (B.22).

(2) For $0 \leq \tau<\infty$ and by using (B.10), (B.15), (B.16), and (B.18) double integral in (B.8) can be similarly derived and expressed as in the previous case. But in this case the boundaries $v_{5}, v_{6}, v_{7}$, and $v_{8}$ which are in (B.15) and (B.16) are used. Then double integral in (B.8) is as follows:

$$
\begin{aligned}
& \iint_{t}^{t+|\tau|} \Phi_{\nu \nu}(\alpha-\beta) d \alpha d \beta \\
& \quad=\int_{-|\tau|}^{|\tau|}(|\tau|-|u|) \Phi_{\nu \nu}(u) d u .
\end{aligned}
$$


By substituting results (B.22) and (B.20) to (B.7) and (B.8) and by merging those results together we obtain for $-\infty<\tau<\infty$ (14):

$$
\begin{aligned}
& E\left\{\left[\theta_{E}(t+\tau)-\theta_{E}(t)\right]^{2}\right\} \\
& =4 \pi^{2} \int_{-|\tau|}^{|\tau|}(|\tau|-|u|) \Phi_{\nu v}(u) d u, \\
& \iint_{t-|\tau|}^{t} \Phi_{\nu v}(\alpha-\beta) d \alpha d \beta \\
& =\iint_{O(\alpha, \beta)} \Phi_{\nu \nu}(\alpha-\beta) d \alpha d \beta \\
& =\iint_{O(u, v)} \Phi_{\nu v}(u) R d v d u \\
& =\int_{-|\tau|}^{0}\left[R \int_{v_{1}}^{v_{2}} 1 d v\right] \Phi_{\nu v}(u) d u \\
& +\int_{0}^{|\tau|}\left[R \int_{v_{3}}^{v_{4}} 1 d v\right] \Phi_{\nu v}(u) d u \\
& =\int_{-|\tau|}^{0}(|\tau|+u) \Phi_{\nu v}(u) d u \\
& +\int_{0}^{|\tau|}(|\tau|-u) \Phi_{\nu v}(u) d u \\
& =\int_{-|\tau|}^{0}(|\tau|-|u|) \Phi_{\nu v}(u) d u \\
& +\int_{0}^{|\tau|}(|\tau|-|u|) \Phi_{\nu v}(u) d u \\
& =\int_{-|\tau|}^{|\tau|}(|\tau|-|u|) \Phi_{\nu \nu}(u) d u .
\end{aligned}
$$

\section{Competing Interests}

The authors declare that there is no conflict of interests regarding the publication of this paper.

\section{Acknowledgments}

This work was supported by the Slovak Research and Development Agency (APVV-0025-12). The research is supported by the European Regional Development Fund and the Slovak state budget for the project "Research Centre of University of Zilina,” ITMS 26220220183.

\section{References}

[1] P. J. Winzer, "High-spectral-efficiency optical modulation formats," Journal of Lightwave Technology, vol. 30, no. 24, pp. 38243835, 2012.

[2] F. Idachaba, D. U. Ike, and O. Hope, "Future trends in fiber optics communication," in Proceedings of the in Proceedings of the World Congress on Engineering, vol. I, pp. 2-6, 2014.
[3] R. Zeleny and M. Lucki, "Nearly zero dispersion-flattened photonic crystal fiber with fluorine-doped three-fold symmetry core," Optical Engineering, vol. 52, no. 4, Article ID 045003, 2013.

[4] A. J. C. Davidson, I. Glesk, and A. Buis, "Has silicon reached it's limit?" Advances in Electrical and Electronic Engineering, vol. 12, no. 6, pp. 590-598, 2015.

[5] R. Agalliu and M. Lucki, "Benefits and limits of modulation formats for optical communications," Advances in Electrical and Electronic Engineering, vol. 12, no. 2, pp. 160-167, 2014.

[6] I. Fatadin, D. Ives, and S. J. Savory, "Laser linewidth tolerance for 16-QAM coherent optical systems using QPSK partitioning," IEEE Photonics Technology Letters, vol. 22, no. 9, article no. 16, pp. 631-633, 2010.

[7] S. T. Naimi, S. Ó. Dúill, and L. P. Barry, "Simulations of the OSNR and laser linewidth limits for reliable wavelength conversion of DQPSK signals using four-wave mixing," Optics Communications, vol. 310, pp. 150-155, 2014.

[8] T. N. Huynh, L. Nguyen, and L. P. Barry, "Delayed selfheterodyne phase noise measurements with coherent phase modulation detection," IEEE Photonics Technology Letters, vol. 24, no. 4, pp. 249-251, 2012.

[9] J. Litvik, D. Benedikovic, J. Dubovan, and M. Kuba, "Numerical investigation of noise chracteristics of telecommunication laser sources for various modulation formats," in Proceedings of the Optical Modelling and Design III, vol. 9131 of Proceedings of SPIE, Brussels, Belgium, April 2014.

[10] J. Litvik, J. Dubovan, D. Benedikovic, and M. Kuba, "Impact of impulse response of avalanche photodiode to eye-closure penalty," in Proceedings of the 38th IEEE International Conference on Telecommunications and Signal Processing (TSP '15), pp. 1-4, Prague, Czech Republic, July 2015.

[11] R. J. Fronen, "Correlation between 1/f fluctuations in the two output beams of a laser diode," IEEE Journal of Quantum Electronics, vol. 27, no. 4, pp. 931-936, 1991.

[12] C. H. Henry, "Theory of the linewidth of semiconductor lasers," IEEE Journal of Quantum Electronics, vol. 18, no. 2, pp. 259-264, 1982.

[13] K. Kikuchi, "Effect of 1/f-type FM noise on semiconductorlaser linewidth residual in high-power limit," IEEE Journal of Quantum Electronics, vol. 25, no. 4, pp. 684-688, 1989.

[14] K. Kikuchi and T. Okoshi, "Estimation of linewidth enhancement factor of AlGaAs lasers by correlation measurement between FM and AM noises," IEEE Journal of Quantum Electronics, vol. 21, no. 6, pp. 669-673, 1985.

[15] L. B. Mercer, "1/f Frequency noise effects on self-heterodyne linewidth measurements," Journal of Lightwave Technology, vol. 9, no. 4, pp. 485-493, 1991.

[16] N. Bucalovic, V. Dolgovskiy, C. Schori, P. Thomann, G. Di Domenico, and S. Schilt, "Experimental validation of a simple approximation to determine the linewidth of a laser from its frequency noise spectrum," Applied Optics, vol. 51, no. 20, pp. 4582-4588, 2012.

[17] G. Di Domenico, S. Schilt, and P. Thomann, "Simple approach to the relation between laser frequency noise and laser line shape," Applied Optics, vol. 49, no. 25, pp. 4801-4807, 2010.

[18] T. Duthel, G. Clarici, C. R. S. Fludger, J. C. Geyer, C. Schulien, and S. Wiese, "Laser linewidth estimation by means of coherent detection," IEEE Photonics Technology Letters, vol. 21, no. 20, pp. 1568-1570, 2009.

[19] M. A. Arvizu and J. M. Francisco, "Rice representation of noise processes in optical PLL's, in AIP Conference Proceedings, vol. 780, no. 1, pp. 385-388, AIP, Salamanca, Spain, 2005. 
[20] D. S. Elliott, R. Roy, and S. J. Smith, "Extracavity laser bandshape and bandwidth modification," Physical Review A, vol. 26, no. 1, pp. 12-18, 1982.

[21] I. Fatadin, D. Ives, and M. Wicks, "Numerical simulation of intensity and phase noise from extracted parameters for CW DFB lasers," IEEE Journal of Quantum Electronics, vol. 42, no. 9, pp. 934-941, 2006.

[22] M. Ahmed, M. Yamada, and M. Saito, "Numerical modeling of intensity and phase noise in semiconductor lasers," IEEE Journal of Quantum Electronics, vol. 37, no. 12, pp. 1600-1610, 2001.

[23] L. E. Richter, H. I. Mandelberg, M. S. Kruger, and P. A. McGrath, "Linewidth determination from self-heterodyne measurements with subcoherence delay times," IEEE Journal of Quantum Electronics, vol. 22, no. 11, pp. 2070-2074, 1986.

[24] P. B. Gallion and G. Debarge, "Quantum phase noise and field correlation in single frequency semiconductor laser systems," IEEE Journal of Quantum Electronics, vol. 20, no. 4, pp. 343-349, 1984.

[25] M. Ahmed, “Theoretical modeling of intensity noise in InGaN semiconductor lasers," Scientific World Journal, vol. 2014, Article ID 475423, 6 pages, 2014.

[26] H. O. Di Roccoa and A. Cruzado, "The voigt profile as a sum of a gaussian and a lorentzian functions, when the weight coefficient depends only on the widths ratio," Acta Physica Polonica A, vol. 122, no. 4, pp. 666-669, 2012.

[27] P. D. Lax, "Change of variables in multiple integrals," The American Mathematical Monthly, vol. 106, no. 6, pp. 497-501, 1999. 


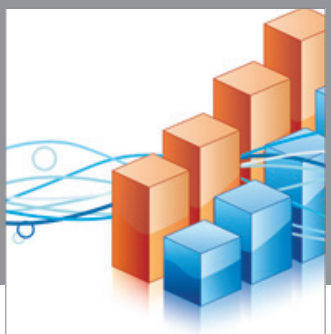

Advances in

Operations Research

vatem alat4

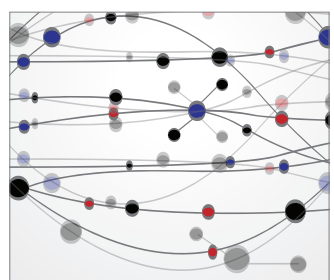

\section{The Scientific} World Journal
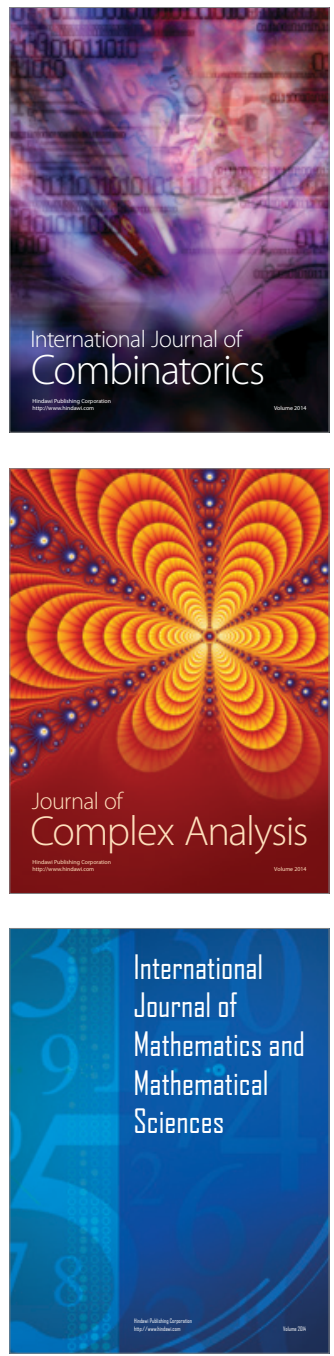
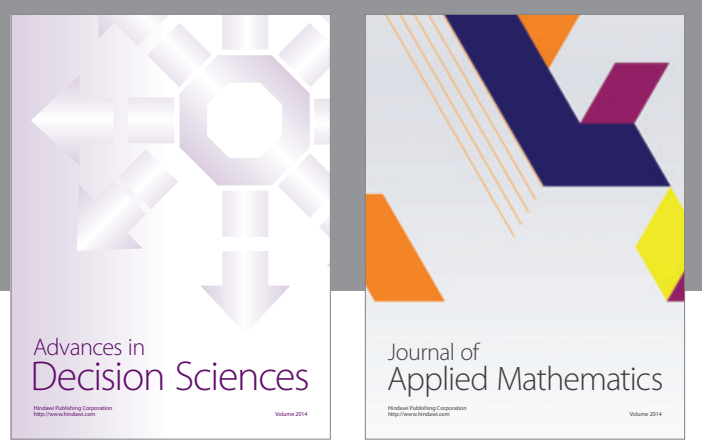

Algebra

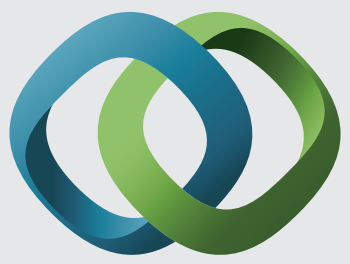

\section{Hindawi}

Submit your manuscripts at

http://www.hindawi.com
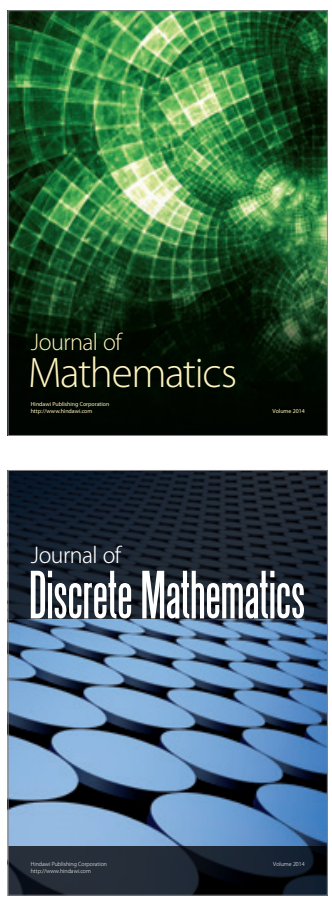

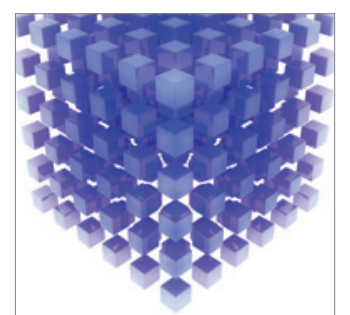

Mathematical Problems in Engineering
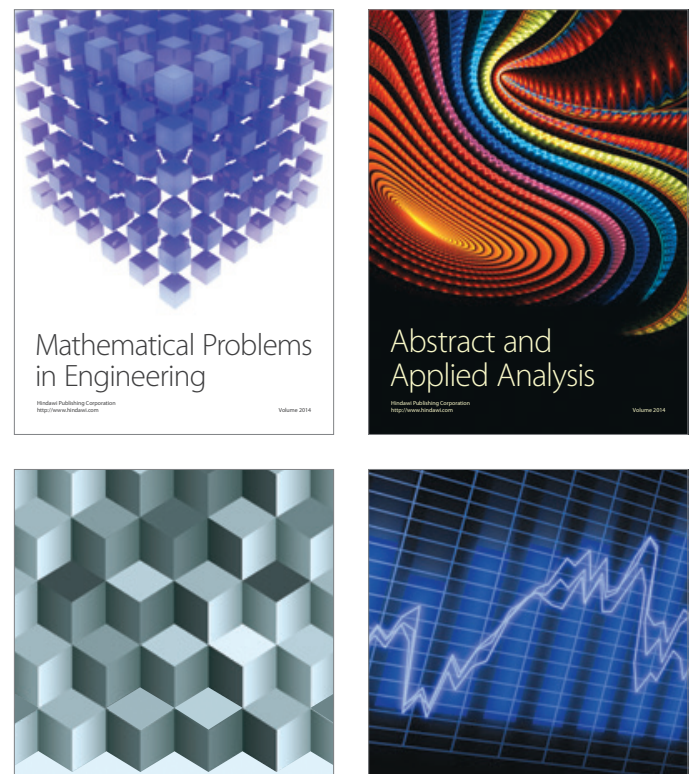

Journal of

Function Spaces

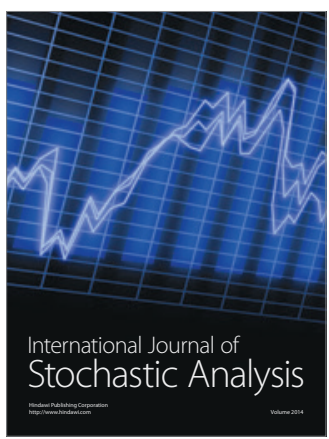

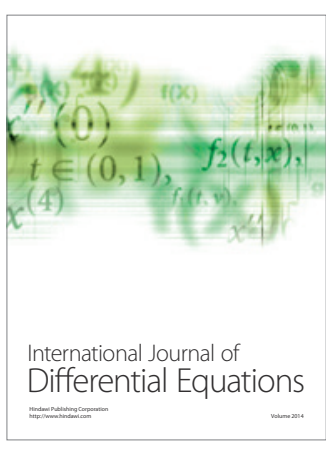
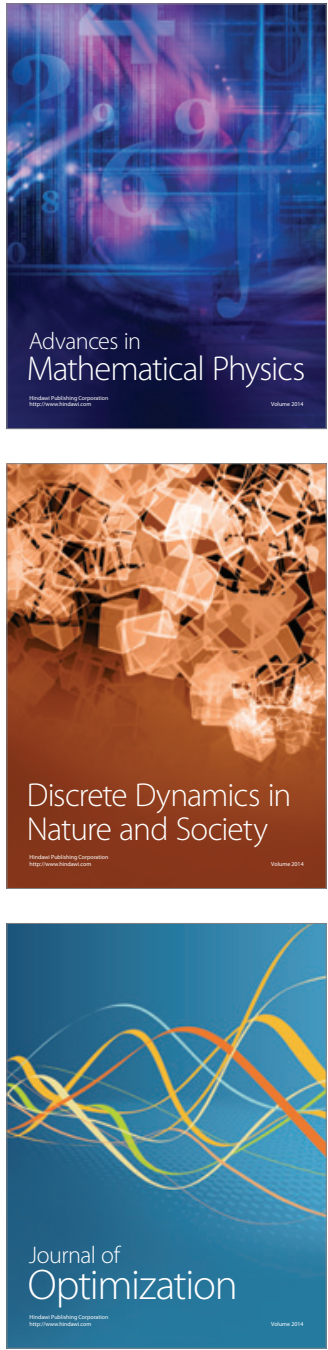